PROCEEDINGS OF THE

AMERICAN MATHEMATICAL SOCIETY

Volume 134, Number 10, October 2006, Pages 2839-2848

S 0002-9939(06)08319-5

Article electronically published on April 11, 2006

\title{
A REMARK ON THE KOCHEN-SPECKER THEOREM AND SOME CHARACTERIZATIONS OF THE DETERMINANT ON SETS OF HERMITIAN MATRICES
}

\author{
LAJOS MOLNÁR \\ (Communicated by Joseph A. Ball)
}

\begin{abstract}
In this paper we describe the general forms of all (nonlinear) continuous functionals on the sets of positive definite, positive semi-definite and Hermitian matrices which are multiplicative on the commuting elements. As a consequence, we obtain some new characterizations of the determinant on those classes of matrices.
\end{abstract}

\section{INTRODUCTION AND STATEMENTS OF THE RESULTS}

The aim of this paper is twofold. On one hand, our investigations have been motivated by the famous Kochen-Specker theorem concerning the problem of hidden variables in quantum mechanics. The physical content of this result is the impossibility of a supplementation of quantum mechanics through noncontextual hidden variables, while its mathematical formulation reads as follows. Let $H$ be a complex Hilbert space with $\operatorname{dim} H \geq 3$ and let $B_{s}(H)$ denote the Jordan algebra of all self-adjoint bounded linear operators on $H$. The Kochen-Specker theorem [6] states that there is no such function $\phi: B_{s}(H) \rightarrow \mathbb{R}$ which satisfies the following conditions:

(a) $\phi(I)=1$;

(b) $\phi(\lambda A)=\lambda \phi(A)$;

(c) $\phi(A+B)=\phi(A)+\phi(B)$ if $A B=B A$;

(d) $\phi(A B)=\phi(A) \phi(B)$ if $A B=B A$

for every $A, B \in B_{s}(H)$ and $\lambda \in \mathbb{R}$. So, roughly speaking, the theorem states that there is no nontrivial real functional on $B_{s}(H)$ which is linear and multiplicative on the commuting pairs of operators in $B_{s}(H)$. Our purpose here is to investigate "the multiplicative part" of the problem separately. In what follows we show that there do exist and, in the case of finite-dimensional spaces, we describe the general form of all such continuous functionals on $B_{s}(H)$ which are multiplicative on the commuting elements. Our approach begins with the consideration of the problem

Received by the editors February 9, 2005 and, in revised form, April 26, 2005.

2000 Mathematics Subject Classification. Primary 15A60, 15A15; Secondary 15A48, 15A57.

Key words and phrases. Positive definite matrices, positive semi-definite matrices, Hermitian matrices, Kochen-Specker theorem, determinant, Jordan triple product.

The author was supported by the Hungarian National Foundation for Scientific Research (OTKA), Grant No. T043080, T046203, and by a joint Hungarian-Slovene grant (Reg. No. SLO-10/03).

(C)2006 American Mathematical Society Reverts to public domain 28 years from publication 
on the positive definite matrices. Then we go to the positive semi-definite matrices and finally to all Hermitian matrices.

On the other hand, parallel to this investigation, we obtain characterizations of the determinant on the above sets of matrices. There are several characterizations of the determinant in the literature. The theorem which is probably the closest one to our present results is due to D. Fearnley-Sander [4. It states that on the algebra of all $n \times n$ real or complex matrices the determinant is the unique multiplicative functional which satisfies certain natural normalizing conditions. (For further interesting results we refer to the papers [1] and [3.) In what follows we obtain results that characterize the determinant not on the whole matrix space but some of its undoubtedly important parts which have been mentioned above. The key property in our characterizations is a kind of multiplicativity which fits the structures of matrices that we consider. In fact, these sets of matrices are not closed under the usual operator theoretical multiplication, but it is easy to see that they are closed under the so-called Jordan triple product $(A, B) \mapsto A B A$ whose operation comes from ring theory. We shall see below that multiplicativity with respect to this product characterizes the determinant on the mentioned sets of matrices rather firmly.

Let us fix the notation. Let $P D_{n}, P S D_{n}$ and $H_{n}$ denote the sets of all $n \times n$ complex positive definite, positive semi-definite and Hermitian matrices, respectively. In what follows, we suppose that $n \geq 3$.

We now turn to the formulation of our results. The first theorem describes the general form of all continuous real-valued functionals on positive definite matrices which are multiplicative on the commuting elements.

Theorem 1. Let $\phi: P D_{n} \rightarrow \mathbb{R}$ be a continuous function with the property that for any $A, B \in P D_{n}$ with $A B=B A$ we have $\phi(A B)=\phi(A) \phi(B)$. Then either

$$
\phi(A)=0 \quad\left(A \in P D_{n}\right)
$$

or there exists a self-adjoint matrix $T$ such that

$$
\phi(A)=\exp (\operatorname{tr}(T \ln A)) \quad\left(A \in P D_{n}\right) .
$$

Corresponding to the above result we obtain the following characterization of the determinant on the set of positive definite matrices.

Theorem 2. Let $\phi: P D_{n} \rightarrow \mathbb{R}$ be a continuous function which satisfies

$$
\phi(A B A)=\phi(A) \phi(B) \phi(A) \quad\left(A, B \in P D_{n}\right) .
$$

Then either

$$
\phi(A)=0 \quad\left(A \in P D_{n}\right)
$$

or there exists a number $c \in \mathbb{R}$ and $u \in\{-1,1\}$ such that

$$
\phi(A)=u(\operatorname{det} A)^{c} \quad\left(A \in P D_{n}\right) .
$$

From our first two theorems we easily obtain the following results for functionals on the set of positive semi-definite matrices.

Corollary 1. Let $\phi: P S D_{n} \rightarrow \mathbb{R}$ be a continuous function with the property that for any $A, B \in P S D_{n}$ with $A B=B A$ we have $\phi(A B)=\phi(A) \phi(B)$. Then we have the following three possibilities:

(i) $\phi(A)=0 \quad\left(A \in P S D_{n}\right)$;

(ii) $\phi(A)=1 \quad\left(A \in P S D_{n}\right)$; 
(iii) there exists a positive definite matrix $T$ such that

$$
\phi(A)= \begin{cases}\exp (\operatorname{tr}(T \ln A)), & \text { if } A \in P S D_{n} \text { is nonsingular } \\ 0, & \text { if } A \in P S D_{n} \text { is singular. }\end{cases}
$$

Corollary 2. Let $\phi: P S D_{n} \rightarrow \mathbb{R}$ be a continuous function which satisfies

$$
\phi(A B A)=\phi(A) \phi(B) \phi(A) \quad\left(A, B \in P S D_{n}\right) .
$$

Then we have the following possibilities:

(i) $\phi(A)=0\left(A \in P S D_{n}\right)$;

(ii) $\phi(A)=u\left(A \in P S D_{n}\right)$ for some $u \in\{-1,1\}$;

(iii) there exists a real number $c>0$ and $u \in\{-1,1\}$ such that

$$
\phi(A)=u(\operatorname{det} A)^{c} \quad\left(A \in P S D_{n}\right) .
$$

Finally, we turn to the case when the domain of our functionals is the whole set of Hermitian matrices. For any $A \in H_{n}$, denote by $i_{-}(A)$ the number of negative eigenvalues of $A$ counted according to multiplicity. The following theorem presents the complete description of all continuous real-valued functionals on $H_{n}$ which are multiplicative on the commuting elements.

Theorem 3. Let $\phi: H_{n} \rightarrow \mathbb{R}$ be a continuous function with the property that for any $A, B \in H_{n}$ with $A B=B A$ we have $\phi(A B)=\phi(A) \phi(B)$. Then we have the following four possibilities:

(i) $\phi(A)=0 \quad\left(A \in H_{n}\right)$;

(ii) $\phi(A)=1 \quad\left(A \in H_{n}\right)$;

(iii) there exists a positive definite matrix $T$ such that

$$
\phi(A)= \begin{cases}\exp (\operatorname{tr}(T \ln |A|)), & \text { if } A \in H_{n} \text { is nonsingular; } \\ 0, & \text { if } A \in H_{n} \text { is singular; }\end{cases}
$$

(iv) there exists a positive definite matrix $T$ such that

$$
\phi(A)= \begin{cases}(-1)^{i_{-}(A)} \exp (\operatorname{tr}(T \ln |A|)), & \text { if } A \in H_{n} \text { is nonsingular; } \\ 0, & \text { if } A \in H_{n} \text { is singular. }\end{cases}
$$

The corresponding characterization of the determinant is formulated in the next theorem for which we need the following notation. Let $H_{n}^{-1}$ denote the set of all invertible elements of $H_{n}$. For any $k=0,1, \ldots, n$, denote $H_{n}^{-1}(k)$ as the set of elements of $H_{n}^{-1}$ which have exactly $k$ negative eigenvalues. It is interesting to observe that, as the following result shows, the behaviour of the continuous functions $\phi: H_{n} \rightarrow \mathbb{R}$ satisfying the equation $\phi(A B A)=\phi(A) \phi(B) \phi(A)$ can be "wider" from a certain point of view than that of the ones which are multiplicative on the commuting elements.

Theorem 4. Let $\phi: H_{n} \rightarrow \mathbb{R}$ be a continuous function which satisfies

$$
\phi(A B A)=\phi(A) \phi(B) \phi(A) \quad\left(A, B \in H_{n}\right) .
$$

Then we have the following possibilities:

(i) $\phi(A)=0\left(A \in H_{n}\right)$;

(ii) $\phi(A)=u\left(A \in H_{n}\right)$ for some $u \in\{-1,1\}$;

(iii) there exists a positive real number $c>0$ and $u_{k} \in\{-1,1\}(k=0, \ldots, n)$ such that

$$
\phi(A)= \begin{cases}u_{k}|\operatorname{det} A|^{c}, & \text { if } A \in H_{n}^{-1}(k) \\ 0, & \text { if } A \in H_{n} \text { is singular. }\end{cases}
$$


We shall close the series of our results with the following rather easy statement. As noted above, the set of all Hermitian matrices is not closed under the usual operator product. This was the reason to consider the so-called Jordan triple product. But, in contrast with the case of positive definite or positive semi-definite matrices, the set of Hermitian matrices is closed under another product, namely, the wellknown Jordan product which plays so important a role in the theory of operator algebras as well as in the mathematical descriptions of quantum mechanics. The Jordan product of $A, B \in H_{n}$ is defined as $(A B+B A) / 2$. Concerning the connection between these two products (we mean the Jordan product and Jordan triple product), we mention the following well-known result, the proof of which requires only an elementary and short calculation. Every linear transformation between algebras which is a homomorphism with respect to the Jordan product is necessarily a homomorphism with respect to the Jordan triple product and, conversely, if the algebras are unital and the linear transformation we consider maps the unit to unit, then the converse statement is also true. However, in contrast with Theorem 4 we have the following easy result.

Proposition 1. Let $\phi: H_{n} \rightarrow \mathbb{R}$ be a continuous function which satisfies

$$
\phi((A B+B A) / 2)=\phi(A) \phi(B) \quad\left(A, B \in H_{n}\right) .
$$

Then $\phi$ is either identically 0 or identically 1.

We remark that the reason for this big difference in the conclusion clearly lies in the fact that this last equation also involves a kind of additivity besides multiplicativity.

\section{Proofs}

We begin with the following easy lemma which is certainly well known. We present it with proof because it is so simple and nice. In what follows, let $\mathbb{R}_{+}$stand for the set of all positive real numbers.

Lemma 1. Let $f: \mathbb{R}_{+} \rightarrow \mathbb{R}_{+}$be a continuous multiplicative function. Then there is a constant $c \in \mathbb{R}$ such that $f(t)=t^{c}\left(t \in \mathbb{R}_{+}\right)$.

Proof. Consider the function

$$
F(s)=\ln f\left(e^{s}\right) \quad(s \in \mathbb{R}) .
$$

Clearly, this is a continuous additive function on $\mathbb{R}$ which is then linear and hence a scalar multiple of the identity. Going back to the original function $f$, we easily obtain the assertion.

Our approach to the proofs of the results of the paper is basically functional analytical. Therefore, in what follows, instead of working with matrices, we consider linear operators acting on a (finite) $n$-dimensional complex Hilbert space $H$.

Proof of Theorem 1. First we prove that the range of $\phi$ lies in the set of nonnegative real numbers. Let $A \in P D_{n}$. We compute

$$
\phi(A)=\phi(\sqrt{A} \sqrt{A})=\phi(\sqrt{A}) \phi(\sqrt{A}) \geq 0 .
$$

Now, suppose that $\phi\left(A_{0}\right)=0$ for some $A_{0} \in P D_{n}$. Then we obtain $\phi(I)=$ $\phi\left(A_{0} A_{0}^{-1}\right)=\phi\left(A_{0}\right) \phi\left(A_{0}^{-1}\right)=0$. This implies

$$
\phi(A)=\phi(I A)=\phi(I) \phi(A)=0
$$


for every $A \in P D_{n}$. So, in what follows we can assume that $\phi$ is everywhere positive.

Pick any projection $P$ on $H$ and denote $P^{\perp}=I-P$. Consider the function

$$
\lambda \longmapsto \phi\left(\lambda P+P^{\perp}\right) .
$$

Clearly, it is a continuous multiplicative function on $\mathbb{R}_{+}$. Therefore, by Lemma 1 we have a real number $\alpha(P) \in \mathbb{R}$ such that

$$
\phi\left(\lambda P+P^{\perp}\right)=\lambda^{\alpha(P)} \quad\left(\lambda \in \mathbb{R}_{+}\right) .
$$

Let $P, Q$ be mutually orthogonal projections on $H$. Then, on one hand, we have

$$
\phi\left(\lambda(P+Q)+(P+Q)^{\perp}\right)=\lambda^{\alpha(P+Q)} .
$$

But, on the other hand, using the commutativity of the operators $\lambda P+P^{\perp}$ and $\lambda Q+Q^{\perp}$, we compute

$$
\begin{gathered}
\phi\left(\lambda(P+Q)+(P+Q)^{\perp}\right)=\phi\left(\left(\lambda P+P^{\perp}\right)\left(\lambda Q+Q^{\perp}\right)\right) \\
=\phi\left(\lambda P+P^{\perp}\right) \phi\left(\lambda Q+Q^{\perp}\right)=\lambda^{\alpha(P)+\alpha(Q)} \quad\left(\lambda \in \mathbb{R}_{+}\right) .
\end{gathered}
$$

Therefore, we obtain that $\alpha(P+Q)=\alpha(P)+\alpha(Q)$ holds for all mutually orthogonal projections $P, Q$ on $H$. In other words, $\alpha$ is an orthogonally additive $\mathbb{R}$-valued function on the set of all projections on $H$. By the continuity of $\phi$ we have the continuity of $\alpha$. Since the set of all projections on $H$ is compact in the norm topology, we obtain that $\alpha$ is bounded. By a variant of Gleason's theorem (see, for example, [2, Theorem 3.2.16]) there exists a self-adjoint linear operator $T$ on $H$ such that

$$
\alpha(P)=\operatorname{tr} T P
$$

holds for every projection $P$ on $H$. Pick an $A \in P D_{n}$ and consider its spectral resolution

$$
A=\lambda_{1} P_{1}+\ldots+\lambda_{n} P_{n}
$$

where the $\lambda_{k}$ 's are positive real numbers and the $P_{k}$ 's are mutually orthogonal rank-one projections. We compute

$$
\begin{aligned}
\phi(A)= & \phi\left(\left(\lambda_{1} P_{1}+P_{1}^{\perp}\right) \cdots\left(\lambda_{n} P_{n}+P_{n}^{\perp}\right)\right)=\phi\left(\lambda_{1} P_{1}+P_{1}^{\perp}\right) \cdots \phi\left(\lambda_{n} P_{n}+P_{n}^{\perp}\right) \\
= & \lambda_{1}^{\operatorname{tr} T P_{1}} \cdots \lambda_{n}^{\operatorname{tr} T P_{n}}=\exp \left(\operatorname{tr} T P_{1} \ln \lambda_{1}+\ldots+\operatorname{tr} T P_{n} \ln \lambda_{n}\right) \\
= & \exp \left(\operatorname{tr} T\left(\ln \lambda_{1} P_{1}+\ldots+\ln \lambda_{n} P_{n}\right)\right)=\exp (\operatorname{tr}(T \ln A)) .
\end{aligned}
$$

This completes the proof.

Proof of Theorem 2. Observe that $\phi(I)=\phi(I)^{3}$, which implies that $\phi(I)$ is 0 , or 1 , or -1 . In the first case we easily obtain that $\phi$ is identically 0 . For the remaining cases, multiplying $\phi$ by -1 if necessary, we can suppose without loss of generality that $\phi(I)=1$.

We prove that $\phi$ is multiplicative on the commuting elements. If $A, B \in P D_{n}$ are commuting, then we have

$$
\phi(A B)=\phi(\sqrt{A} B \sqrt{A})=\phi(\sqrt{A}) \phi(B) \phi(\sqrt{A}) .
$$

On the other hand, we compute

$$
\phi(\sqrt{A}) \phi(\sqrt{A})=\phi(\sqrt{A}) \phi(I) \phi(\sqrt{A})=\phi(A),
$$

which implies $\phi(\sqrt{A})=\sqrt{\phi(A)}$. Going back to the previous equality, we get that

$$
\phi(A B)=\sqrt{\phi(A)} \phi(B) \sqrt{\phi(A)}=\phi(A) \phi(B) .
$$


By Theorem 1 we obtain that there is a self-adjoint linear operator $T$ on $H$ such that

$$
\phi(A)=\exp (\operatorname{tr}(T \ln A)) \quad\left(A \in P D_{n}\right) .
$$

In the rest of the proof we show that $T$ is a scalar multiple of the identity.

By the multiplicativity property and the form (11) of $\phi$ we have the following equality:

$$
\operatorname{tr}(T \ln (A B A))=\operatorname{tr}(T \ln A)+\operatorname{tr}(T \ln B)+\operatorname{tr}(T \ln A) \quad\left(A, B \in P D_{n}\right) .
$$

Pick projections $P, Q$ on $H$. Let

$$
A=I+t P, \quad B=I+t Q,
$$

where $t>-1$ is any real number. An easy computation shows that

$A B A=(I+t P)(I+t Q)(I+t P)=I+t(2 P+Q)+t^{2}(P+P Q+Q P)+t^{3}(P Q P)$.

It is known that in an arbitrary unital Banach algebra $\mathcal{A}$, for any $a \in \mathcal{A}$ with $\|a\|<1$ we have

$$
\ln (1+a)=\sum_{n=1}^{\infty} \frac{(-1)^{n+1} a^{n}}{n}
$$

(see, e.g., [7, 3.4.4. Theorem]). This shows that for a suitable positive $\epsilon$, the operators $\ln (A B A), \ln A, \ln B$ can be expressed by power series of $t(|t|<\epsilon)$ with operator coefficients. Considering the coefficients of $t^{3}$ on both sides of the equality (21) and using the uniqueness of the coefficients, we obtain the following equation:

$$
\begin{aligned}
\operatorname{tr} T\left(P Q P-\frac{1}{2}((2 P+Q)(P+P Q+Q P)+(P+P Q+Q P)(2 P+Q))\right. & \\
\left.+\frac{1}{3}(2 P+Q)^{3}\right) & =\operatorname{tr} T\left(\frac{1}{3}(P+Q+P)\right) .
\end{aligned}
$$

Performing the necessary computations and subtracting those terms which appear on both sides of this equation, we arrive at the following simple equation:

$$
\operatorname{tr} T\left(\frac{1}{3}(P Q P)-\frac{1}{3}(Q P Q)\right)=0 .
$$

This implies that

$$
\operatorname{tr} T P Q P=\operatorname{tr} T Q P Q
$$

holds for arbitrary projections $P, Q$ on $H$. Choosing unit vectors $x, y \in H$ and inserting $P=x \otimes x, Q=y \otimes y$ into the previous equation, we obtain

$$
|\langle x, y\rangle|^{2}\langle T x, x\rangle=|\langle x, y\rangle|^{2}\langle T y, y\rangle
$$

which, in case $x, y$ are not orthogonal to each other, implies that $\langle T x, x\rangle=\langle T y, y\rangle$. Next, by the continuity of $T$ we obtain

$$
\langle T x, x\rangle=\langle T y, y\rangle
$$

for arbitrary unit vectors $x, y \in H$. This means that there is a constant $c \in \mathbb{R}$ such that $\langle T x, x\rangle=c$ holds for every unit vector $x \in H$, which plainly implies that

$$
\langle T x, x\rangle=\langle c x, x\rangle \quad(x \in H) .
$$

This gives us that $T=c I$. Finally, from (1) we infer that

$$
\phi(A)=\exp (c \operatorname{tr} \ln A)=(\exp (\operatorname{tr} \ln A))^{c}=(\operatorname{det} A)^{c}
$$

holds for any $A \in P D_{n}$. This completes the proof. 
Proof of Corollary 1, Restrict our transformation $\phi$ onto $P D_{n}$. If it is identically 0 , then by continuity we obtain the form (i).

In what follows we are interested in the behaviour of $\phi$ on the singular elements of $P S D_{n}$. Pick a rank- $(n-1)$ projection $P$ on $H$. By the multiplicativity property of $\phi$ we have $\phi(P)=\phi\left(P^{2}\right)=\phi(P)^{2}$, which gives us that $\phi(P)$ is either 0 or 1 . So, we distinguish two cases.

First, let $\phi(P)=1$. It is easy to see that $P$ can be connected by a continuous curve to any other rank- $(n-1)$ projection within the set of all such projections on $H$. By continuity, it follows that $\phi(Q)=1$ holds for every rank- $(n-1)$ projection $Q$ on $H$. Let $\lambda$ be a nonnegative real number and $R$ a rank-one projection. Then we compute

$$
\begin{gathered}
\phi\left(\lambda R+R^{\perp}\right)=1 \cdot \phi\left(\lambda R+R^{\perp}\right)=\phi\left(R^{\perp}\right) \phi\left(\lambda R+R^{\perp}\right) \\
=\phi\left(R^{\perp}\left(\lambda R+R^{\perp}\right)\right)=\phi\left(R^{\perp}\right)=1 .
\end{gathered}
$$

Since every element of $P S D_{n}$ is the product of commuting elements of the form $\lambda R+R^{\perp}$, it now follows that $\phi$ is identically 1 on $P S D_{n}$, i.e., we obtain the form (ii).

We have to also consider the case when $\phi$ is not identically 0 on $P D_{n}$ but we have $\phi(Q)=0$ for every rank- $(n-1)$ projection $Q$ on $H$. If $A \in P S D_{n}$ is singular, then there is a rank- $(n-1)$ projection $Q$ commuting with $A$ such that $Q A=A$. It follows that

$$
\phi(A)=\phi(Q A)=\phi(Q) \phi(A)=0 .
$$

By Theorem 1, we obtain that there is a self-adjoint operator $T$ such that $\phi$ is of the form which appears in (iii).

It remains to show that $T$ is necessarily positive definite. This will follow from the continuity of $\phi$. Indeed, let

$$
T=\epsilon_{1} P_{1}+\ldots+\epsilon_{n} P_{n}
$$

be the spectral resolution of $T$ where the $\epsilon_{i}$ 's are real numbers and the $P_{i}$ 's are mutually orthogonal rank-one projections. We prove that the $\epsilon_{k}$ 's are in fact all positive. For example let us show it for $\epsilon_{1}$. Consider any positive number $\lambda$ and the positive definite operator $A_{\lambda}=\lambda P_{1}+P_{1}^{\perp}$. If $\lambda \rightarrow 0$, then $A_{\lambda} \rightarrow P_{1}^{\perp}$, and by continuity we obtain that $\phi\left(A_{\lambda}\right) \rightarrow \phi\left(P_{1}^{\perp}\right)=0$. On the other hand, computing the value $\phi\left(A_{\lambda}\right)$ using the form in (iii), it follows easily that

$$
\phi\left(A_{\lambda}\right)=\lambda^{\epsilon_{1}} .
$$

Since this must converge to 0 as $\lambda \rightarrow 0$, we get $\epsilon_{1}>0$. In that way we can show that the eigenvalues of $T$ are all positive, which means that $T$ is positive definite. The proof is completed.

Proof of Corollary 2, Using the continuity of $\phi$, it follows from Theorem 2,

Proof of Theorem 3. Consider the restriction $\phi_{s}$ of $\phi$ onto the set $P S D_{n}$. According to Corollary 11 we have three possibilities.

First suppose that $\phi_{s}$ is identically 0 . Then for any $A \in H_{n}$ we have

$$
\phi(A)^{2}=\phi\left(A^{2}\right)=\phi_{s}\left(A^{2}\right)=0,
$$

which implies that $\phi$ is identically 0 . 
Next suppose that $\phi_{s}$ is identically 1 . Similar to the equality (3) we obtain that $\phi$ always takes one of the values -1 and 1 . By the connectedness of $H_{n}$ and the continuity of $\phi$ it follows that $\phi$ is identically 1 .

Now, assume that $\phi_{s}$ is of the third possible form appearing in Corollary 1. Since the square of a singular element of $H_{n}$ is also singular, just as in (3) we infer that $\phi$ is zero on the singular matrices. Define the real-valued function $\psi$ on the invertible elements of $H_{n}$ in the following way:

$$
\psi(A)=\exp (\operatorname{tr}(T \ln |A|)) .
$$

Once again, as in (3) we compute for any invertible $A \in H_{n}$ that

$$
\begin{gathered}
\phi(A)^{2}=\phi\left(A^{2}\right)=\phi_{s}\left(A^{2}\right)=\exp \left(\operatorname{tr}\left(T \ln A^{2}\right)\right) \\
=\exp \left(\operatorname{tr}\left(T \ln |A|^{2}\right)\right)=\exp (\operatorname{tr}(T 2 \ln |A|))=(\exp (\operatorname{tr}(T \ln |A|)))^{2}=\psi(A)^{2} .
\end{gathered}
$$

This implies that

$$
\phi(A)= \pm \psi(A)
$$

for each invertible $A \in H_{n}$.

It is not hard to see that the components of the topological space $H_{n}^{-1}$ are exactly the sets $H_{n}^{-1}(k)(k=0,1, \ldots, n)$. Using the continuity of $\phi$ and $\psi$ on $H_{n}^{-1}$, it follows that the sign \pm in (44) is stable in every inertia class $H_{n}^{-1}(k)$. Clearly, $\phi$ equals $\psi$ on the positive definite matrices, i.e., on $H_{n}^{-1}(0)$. We now distinguish two cases.

First suppose that $\phi(A)=\psi(A)$ for every $A \in H_{n}^{-1}(1)$. It is easy to see that $\psi$ is multiplicative on the commuting pairs of elements of $H_{n}^{-1}$ and that every $B \in H_{n}^{-1}(2)$ is the product of two commuting elements $A_{1}, A_{2}$ of $H_{n}^{-1}(1)$. This gives us that $\phi(B)=\psi(B)$, i.e., $\phi$ equals $\psi$ on $H_{n}^{-1}(2)$. Next, any $C \in H_{n}^{-1}(3)$ is the product of two commuting elements $A \in H_{n}^{-1}(1)$ and $B \in H_{n}^{-1}(2)$. This gives us that $\phi(C)=\psi(C)$. Going further in the same way, we finally get that $\phi$ coincides with $\psi$ on the whole set $H_{n}^{-1}$. This gives us that $\phi$ is of the form which appears in (iii).

Finally, suppose that $\phi(A)=-\psi(A)$ for every $A \in H_{n}^{-1}(1)$. Then in the same way as above, we obtain that $\phi(B)=\psi(B)$ for every $B \in H_{n}^{-1}(2)$, that $\phi(C)=$ $-\psi(C)$ for every $C \in H_{n}^{-1}(3)$, and so on. Clearly, this leads us to the form (iv) of $\phi$ appearing in the formulation of our theorem, and the proof is completed.

Proof of Theorem 4. Just as in the proof of Theorem 2 we obtain that $\phi(I)$ is 0 , or 1 , or -1 . If $\phi(I)=0$, then by the properties of $\phi$ we immediately have that it is identically 0 . Multiplying $\phi$ by -1 if necessary, we can suppose without loss of generality that $\phi(I)=1$. We compute

$$
\phi(A)^{2}=\phi(A) \phi(I) \phi(A)=\phi(A I A)=\phi\left(A^{2}\right) .
$$

We now apply Corollary 2, If $\phi$ is identically 1 on $P S D_{n}$, then the previous equality together with the continuity of $\phi$ gives us that $\phi$ is identically 1 on the whole set $H_{n}$. So, suppose that there is a real number $c>0$ such that $\phi(A)=(\operatorname{det} A)^{c}$ for every $A \in P S D_{n}$. Once again, by the previous displayed formula we obtain that $\phi(A)^{2}=\left(\operatorname{det} A^{2}\right)^{c}$, i.e.,

$$
\phi(A)= \pm|\operatorname{det} A|^{c}
$$

holds for every $A \in H_{n}$. Similar to the proof of Theorem 3 we see that the sign \pm is stable on every inertia class $H_{n}^{-1}(k)$. This completes the proof. 
To see that the $u_{k}$ 's can really be arbitrary, we refer to the concluding remarks of the paper below.

Proof of Proposition 1. For any projection $P$ on $H$ we have

$$
\phi(P)=\phi((P P+P P) / 2)=\phi(P)^{2}
$$

which implies that $\phi(P)$ is either 0 or 1 . We distinguish two cases. First, if $\phi(I)=0$, then it is plain that $\phi$ is identically 0 . So, consider the second case, i.e., when we have $\phi(I)=1$. For any invertible $A \in H_{n}$ we obtain

$$
1=\phi(I)=\phi\left(\left(A A^{-1}+A^{-1} A\right) / 2\right)=\phi(A) \phi\left(A^{-1}\right),
$$

which implies that $\phi(A) \neq 0$. Therefore, $\phi$ is nowhere zero on $H_{n}^{-1}$. Now consider the following two particular invertible operators:

$$
A=\left(\begin{array}{cc}
1 & 0 \\
0 & -1
\end{array}\right) \oplus I_{n-2}, \quad B=\left(\begin{array}{ll}
0 & 1 \\
1 & 0
\end{array}\right) \oplus I_{n-2},
$$

where $I_{n-2}$ denotes the identity on an $(n-2)$-dimensional subspace. It is easy to see that $(A B+B A) / 2$ is a rank- $(n-2)$ projection. As

$$
\phi((A B+B A) / 2)=\phi(A) \phi(B)
$$

and $\phi(A), \phi(B) \neq 0$, it follows that $\phi$ takes the value 1 on this particular projection. By continuity, we infer that this holds true also for all rank- $(n-2)$ projections. It is easy to see that every rank- $(n-3)$ projection can be written in the form $(P Q+Q P) / 2$ for some (commuting) rank- $(n-2)$ projections. Hence we obtain that $\phi$ is 1 on the set of all rank- $(n-3)$ projections. Hence, going down with rank, we finally obtain that $\phi(0)=1$. Using the equality

$$
\phi(0)=\phi((0 A+A 0) / 2)=\phi(0) \phi(A)
$$

we obtain that $\phi$ is identically 1 , and this completes the proof.

\section{Remarks}

We conclude the paper with some remarks.

First, we should emphasize that the converse statements in all of our results above are also valid. In fact, as for the only nontrivial such statement, item (iii) in Theorem 4. we recall the fact from matrix theory that $A$ and $S A S^{*}$ have the same inertia for any Hermitian matrix $A$ and invertible matrix $S$ (see, for example, [5, 4.5.8. Theorem]).

Next, observe that in order to obtain the above nice forms of our functionals, the assumption of continuity in our theorems and corollaries is essential. In fact, for example, considering any discontinuous additive function $a$ on $\mathbb{R}$ and defining

$$
\psi(A)=\exp (a(\ln (\operatorname{det} A))),
$$

we obtain a discontinuous functional which (on all treated domains) could serve as a counterexample.

Finally, we remark that, due to the use of Gleason's theorem, we have had to suppose that the dimension $n$ is at least 3. However, we suspect that the same conclusions are valid also when $n=2$. It would be interesting to find a proof for this case which would probably give a new approach to the case $n \geq 3$ as well without invoking Gleason's theorem. 


\section{ACKNOWLEDGEMENTS}

The author is thankful to the referees whose remarks helped him to improve the presentation of the paper.

\section{REFERENCES}

1. C.G. Cao, Multiplicative functions of $n \times n$ matrix semigroups over arbitrary fields, J. Math. (Wuhan) 11 (1991), 35-38. MR1139669 (92k:20122)

2. A. Dvurečenskij, Gleason's Theorem and Its Applications, Kluwer Academic Publishers, 1993. MR1256736 (94m:46092)

3. J. Fan, Determinants and multiplicative functionals on quaternion matrices, Linear Algebra Appl. 369 (2003), 193-201. MR1988486 (2004c:15007)

4. D. Fearnley-Sander, A characterization of the determinant, Amer. Math. Monthly 82 (1975), 838-840. MR0399126 (53:2977)

5. R.A. Horn and C.R. Johnson, Matrix Analysis, Cambridge University Press, 1996.

6. S. Kochen and E.P. Specker, The problem of hidden variables in quantum mechanics, J. Math. Mech. 17 (1967), 59-87. MR0219280 (36:2363)

7. T.W. Palmer, Banach Algebras and The General Theory of *-Algebras, Vol. I, Encyclopedia Math. Appl. 49, Cambridge University Press, 1994. MR1270014 (95c:46002)

Institute of Mathematics, University of Debrecen, H-4010 Debrecen, P.O. Box 12, HUNGARY

E-mail address: molnarl@math.klte.hu 